\title{
Effectiveness of FDG PET/CT in Metastatic Infections
}

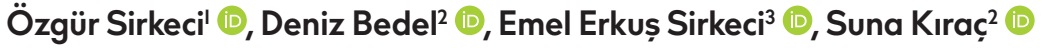 \\ 'Department of Internal Medicine, Near East University Hospital, Nicosia, Cyprus \\ 2Department of Nuclear Medicine, Near East University Hospital, Nicosia, Cyprus \\ ${ }^{3}$ Department of Emergency, Near East University Hospital, Nicosia, Cyprus \\ ORCID IDs of the authors: Ö.S. 0000-000I-9048-5096; D.B. 0000-000I-94I6-6973; E.E.S. 0000-0003-2743-7619; S.K. 0000-0002-044I- \\ 4599.
}

\author{
Cite this article as: Sirkeci Ö, Bedel D, Erkuș sirkeci E, Kıraç S. Effectiveness of FDG PET/CT in Metastatic Infections. Cyprus J Med Sci \\ 2019; 4(1): 66-9.
}

Prosthesis infections that develop two years after the operation or later occur completely hematologically. In some patients, systemic inflammatory response syndrome or metastatic infection can occur. Diagnosing metastatic infections is time consuming and difficult. All the infection foci cannot be found even with the use of many imaging techniques. But F-I8 fluorodeoxyglucose (FDG) positron emission tomography/computed tomography (PET/CT) imaging allows whole body scan anatomically and functionally. Here, we aimed to discuss the role of F-18 FDG PET/CT imaging in detecting and diagnosing multiple abscess foci secondary to Staphylococcus aureus bacteremia in a case with late prosthesis infection and atypical complaints.

Keywords: FDG PET/CT, prosthetic joint infection, pyogenic abscess

\section{INTRODUCTION}

Prosthetic infections are difficult to diagnose and treat, and treatment costs are high. Infections are usually due to polymicrobial, coagulase negative Staphylococci and Staphylococcus aureus (S. aereus). The time since implantation of the prosthesis is of concern. In early prosthetic infections ( $<3$ months), nonspecific complaints such as fever, tachycardia, and joint pain may develop. Infections that develop between 3 and 24 months cause more joint pain. Whereas, infections that develop 2 years after the operation or later occur completely hematologically $(I)$. In some patients, systemic inflammatory response syndrome or metastatic infection can occur. Early detection of metastatic infection enables the identification of appropriate treatment. There are several useful serologic tests and imaging methods for diagnosis. Ultrasonography (USG) and magnetic resonance imaging (MRI) are extremely sensitive and reliable methods to diagnose regional infection foci, but their use is limited in assessing multifocal infection. F-18 fluorodeoxyglucose (FDG) positron emission tomography/computed tomography (PET/CT) imaging has an important role in the detection of multifocal infections. F-18 FDG PET/CT whole body imaging allows examining all organs and tissues from the vertex of head to the foot. It is extremely useful in the detection of infection focus and foci in patients with infection history and predisposing factors such as prosthesis. Here, we aimed to discuss the role of F-18 FDG PET/CT imaging in detecting and diagnosing multiple abscess foci secondary to $S$. aureus bacteremia in a case with late prosthesis infection and atypical complaints.

\section{CASE PRESENTATION}

A 56-year-old female patient was admitted to the outpatient clinic with complaints of right leg swelling and pain, weakness, and high fever. On physical examination, increase in the diameter and temperature, hyperemia, and tenderness were detected in the right leg. Also, left hip joint movements were painful. The patient had a l6-year-old left hip and I2-year-old right hip prosthesis. Doppler ultrasonography showed abscess foci with septations and deep femoral vein thrombosis in the right leg. Treatment was started. During follow-up, increased temperature and hyperemia developed in both wrists, elbows, and right ankle. Hyponatremia and respiratory distress occurred. The CT images showed multiple nodules and ground glass appearance in the lungs. Retroperitoneal lymph nodes were also detected. On the same day, blood cultures were taken because of suddenly rising fever; and atypical pneumonia therapy was started with levofloxacin from the quinolone group. On the second day of treatment, respiratory distress disappeared; 


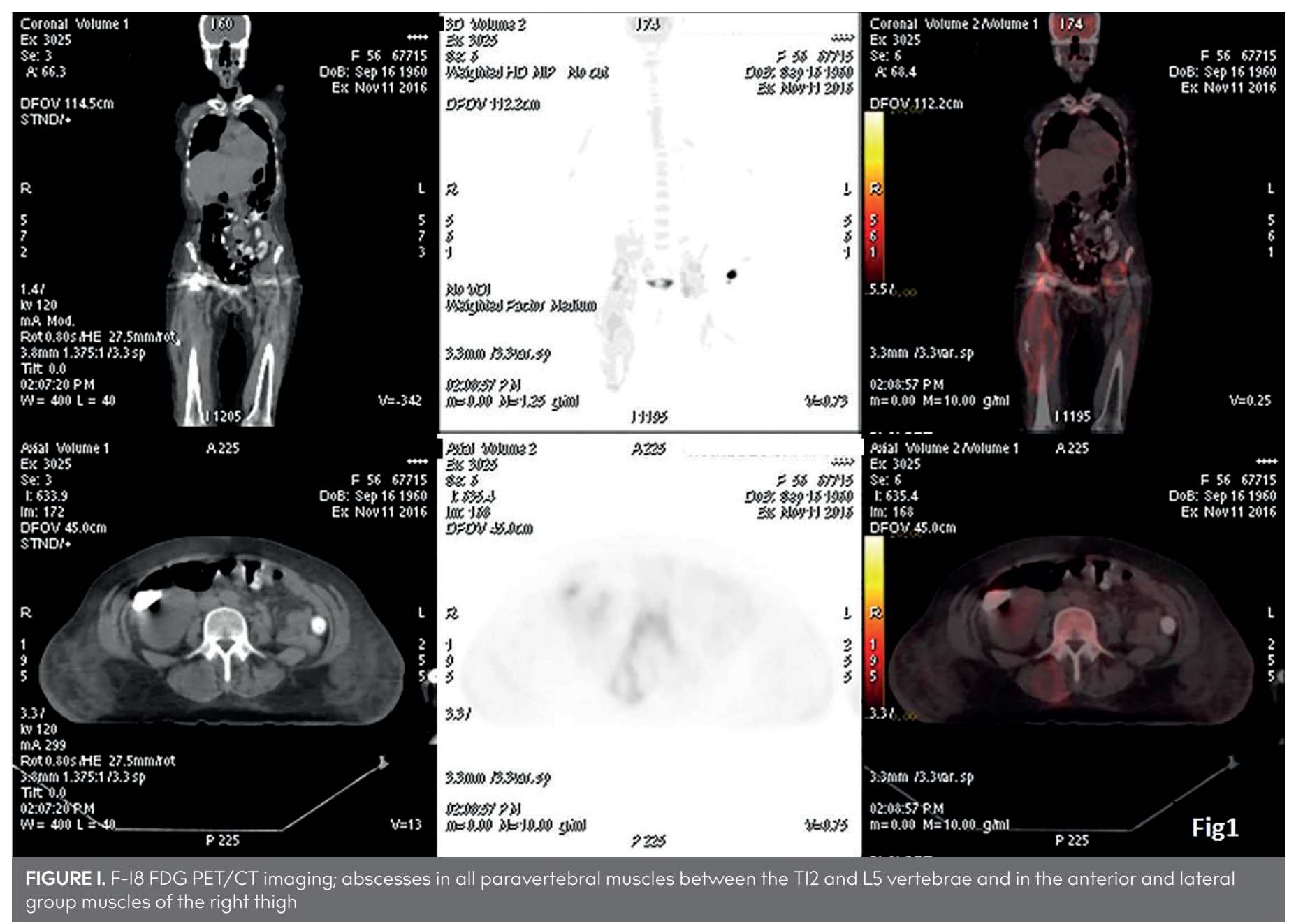

but there was no regression of the arthritis findings, and high fever persisted. On the fifth day of treatment, $S$. aureus was detected in blood cultures; antibiotic therapy was reorganized to teicoplanin from the glycopeptide group. Bilateral hip prostheses are considered to be the source of bacteremia. Hip MRI was performed. It revealed abscesses extending to the femur shaft around the bilateral prosthesis. Surgical treatment was planned for the patient. However, because the patient had pneumonia and multiple arthritis findings, F-18 FDG PET/CT imaging was performed because of the suspicion of bacterial metastatic infection (Figure I). Whole body F-I8 FDG PET/ CT images showed abscesses in all paravertebral muscles between the TI2 and L5 vertebrae and in the anterior and lateral group muscles of the right thigh. Increased F-18 FDG uptake was detected around both hip prostheses (Figure 2). Inflammatory F-18 FDG uptake was observed in the lower extremity veins. However, there was no evidence of active endocarditis in the images.

The abscess foci identified in the F-I8 FDG PET/CT images were drained; and antibiotic therapy continued for 14 days after surgery. Rapid healing was observed in follow-up exams. Arthritis findings improved, hip pain reduced, and high fever was not observed again.

Written informed consent was obtained from the patient who participated in this study.

\section{DISCUSSION}

S. aureus, which may be part of normal flora in some of the healthy people, is a frequently found microorganism in late prosthetic infections. It can cause bacteremia, especially in diabetics, in intravenous drug users, and in those with leukocyte dysfunction. The development of metastatic infection (endocarditis, vasculitis, spondylodiscitis, or pulmonary abscess) is a major complication of gram-positive bacteremia. Early detection of metastatic infection has critical importance. Long-term antibiotic treatment and drainage should be applied in some cases. Metastatic infections can occur in one-third of bacteremia; and they most commonly involve bone, joint, kidney, and lungs. In metastatic infections, the detection of all foci is sometimes impossible; so treatment is often carried out on a determined single focus. In asymptomatic cases, inadequate treatment increases the morbidity and mortality because the infection cannot be eradicated. This situation creates difficulties in treatment.

Whole body F-I8 FDG PET/CT imaging is useful for early and accurate diagnosis of metastatic infection foci in patients with prosthesis and for the detection of focus in the case with fever of unknown origin. F-I8 FDG PET/CT imaging within the first two weeks after detection of bacteremia is beneficial in directing treatment. In their study, Hussman et al. (2) used F-I8 FDG PET/CT imaging following vascular grafts, and reported that it is very valuable in managing the treatment of patients. In the study of Kouijzer IJ et al. (3), focus of infection was identified in $38 \%$ of I 3 pediatric pa- 


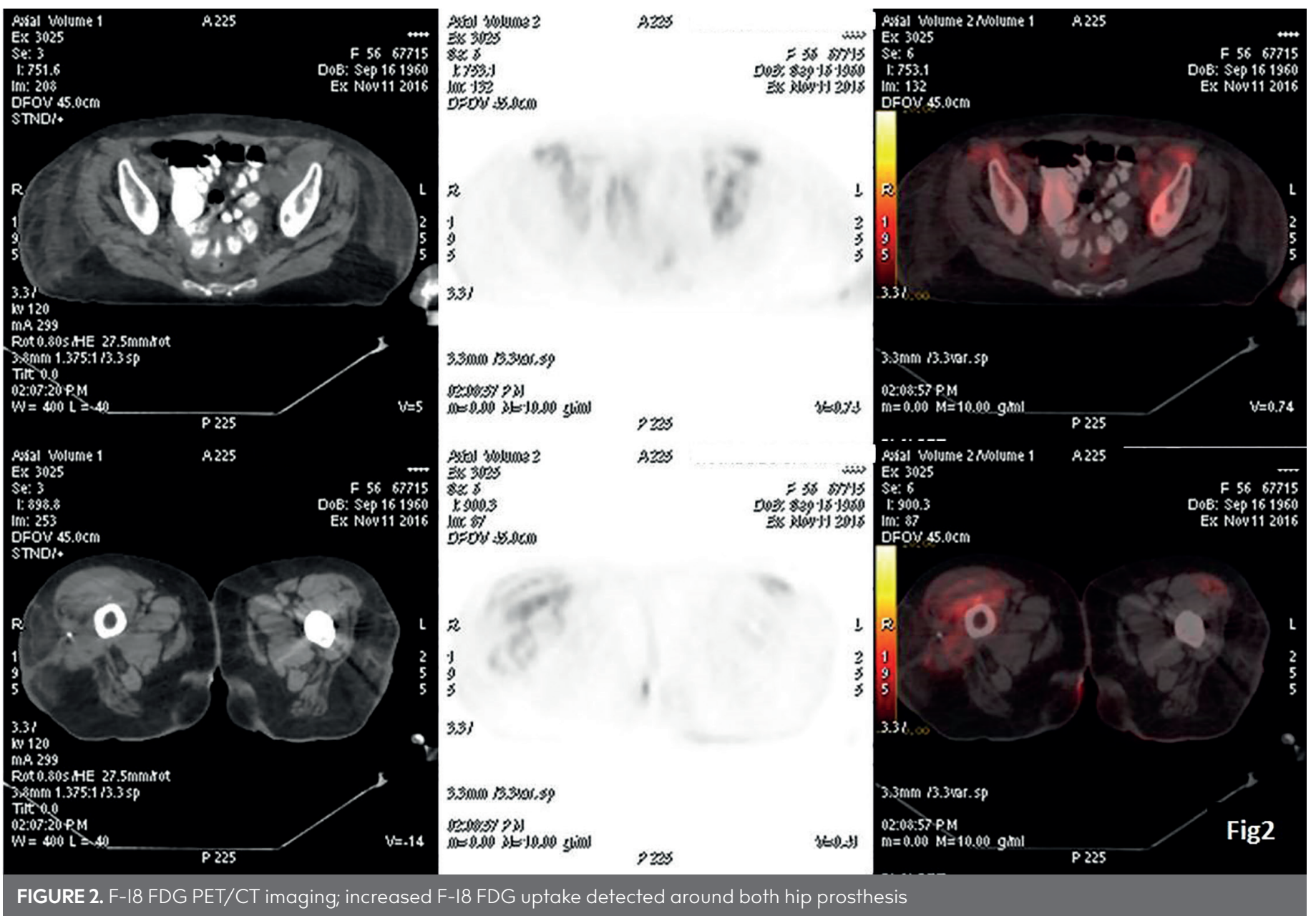

tients who were suspected of metastatic infection. The positive predictive value was calculated as $71 \%$, and the negative predictive value as $100 \%$ (4). Vos FJ et al. (4) reported a sensitivity of $100 \%$, a specificity of $87 \%$, and a negative predictive value of $89 \%$ for F-18 FDG PET/CT imaging in the detection of metastatic infections in patients with gram-positive bacteremia (3).

In our case, presence of multiple infection foci revealed difficulties in treatment planning. We could try to detect infection foci by doing many MR/CT imaging, but this option would be time consuming and expensive. Therefore, we preferred to do F-18 FDG PET/CT imaging; and in this way, we detected all the foci of the infection even also evaluated endocarditis.

F-I8 FDG PET/CT imaging also has an important role in the detection of infection focus in fever of unknown origin. Tseng JR et al. (5) reported that positive FDG PET/CT findings were detected in $35(66 \%)$ of 53 patients with fever of unknown origin; and the treatment of 13 patients was rearranged according to this result. Determining the focus and extent of the disease in metastatic infections is often difficult and time consuming. There are delays in the diagnosis because MRI or CT imaging is performed on too many regions in the patient. This situation negatively affects the prognosis. Whereas, F-I8 FDG PET/CT imaging provides important findings in the determination of foci that cannot be detected by standard examinations, and helps to establish the diagnosis earlier. So, morbidity and mortality decrease. It seems to be more effective in reducing treatment cost and loss of labor since it allows us to choose appropriate therapy (6).

In conclusion, whole body F-I8 FDG PET/CT imaging is useful in the early and accurate diagnosis of metastatic infection foci in patients with prosthesis, and in detecting fever of unknown origin. F-I8 FDG PET/CT imaging performed especially within the first two weeks after detection of bacteremia is of great benefit in directing therapy.

Informed Consent: Written informed consent was obtained from the patient who participated in this study

Peer-review: Externally peer-reviewed.

Author contributions: Concept - O.S., D.B., S.K.; Design - O.S., D.B., E.S.; Supervision - Ö.S., D.B., S.K.; Resource - O.S., D.B., E.S.; Materials - O.S., D.B., E.S.; Data Collection and/or Processing - O.S., D.B., S.K.; Analysis and/or Interpretation - O.S., D.B., S.K.; Literature Search - O.S., D.B., E.S.; Writing O.S., D.B., E.S.; Critical Reviews - O.S., D.B., S.K.

Conflict of Interest: The authors have no conflicts of interest to declare.

Financial Disclosure: The authors declared that this study has received no financial support.

\section{REFERENCES}

I. Diktaș H, Turhan V. Orthopedic Denture Infections: Current Approaches to Diagnosis, Treatment and Management. Mediterr J Infect Microb Antimicrob 2012; I: I-8. 
2. Husmann L, Sah BR, Scherrer A, Burger IA, Stolzmann P, Weber R, et al. ${ }^{18} \mathrm{~F}-F D G$ PET/CT for Therapy Control in Vascular Graft Infections: A First Feasibility Study. VASGRA Cohort. J Nucl Med 2015; 56: 1024-9. [CrossRef]

3. Kouijzer IJ, Blokhuis GJ, Draaisma JM, Oyen WJ, de Geus-Oei LF, Bleeker-Rovers CP. I8F-FDG PET / CT in Detection Metastatic Infection in Children. Clin Nucl Med 2016; 4I: 278-8I. [CrossRef]

4. Vos FJ, Bleeker-Rovers CP, Sturm PD, Krabbe PF, van Dijk AP, Cuijpers $M L$, et al. I8F-FDG PET / CT for detection of metastatic infection in gram-positive bacteremia. J Nucl Med 2010; 5I: 1234-40. [CrossRef]
5. Tseng JR, Lin CW, Chen SH, Yen TH, Lin PY, Lee MH, et al. Clinical Usefulness of FDG PET / CT for Detection of Infections of Unknown Origin in Patients Undergoing Maintenance Hemodialysis. J Nucl Med 2015; 56: 68I-7. [CrossRef]

6. Kouijzer IJ, Vos FJ, Bleeker-Rovers CP, Oyen WJ. Clinical application of FDG-PET / CT in metastatic infections. Q J Nucl Med Mol Imaging 2017; 61: 232-246. 